\title{
ON THE CHERN NUMBERS OF GENERALISED KUMMER VARIETIES
}

\author{
MARC A. NiePER-WisskirChen
}

\begin{abstract}
Let $A^{[[n]]}$ denote the $2(n-1)$-dimensional generalised Kummer variety constructed from the abelian surface $A$. Further, let $X$ be an arbitrary smooth projective surface with $\int_{X} c_{1}(X)^{2} \neq 0$, and $X^{[k]}$ the Hilbert scheme of zerodimensional subschemes of $X$ of length $k$. We give a formula which expresses the value of any complex genus on $A^{[[n]]}$ in terms of Chern numbers of the varieties $X^{[k]}$.

In [4] and [5] it is shown how to use Bott's residue formula to effectively calculate the Chern numbers of the Hilbert schemes $\left(\mathbb{P}^{2}\right)^{[k]}$ of points on the projective plane. Since $\int_{\mathrm{p} 2} c_{1}\left(\mathbb{P}^{2}\right)^{2}=9 \neq 0$, we can use these numbers and our formula to calculate the Chern numbers of the generalised Kummer varieties.

A table with all Chern numbers of the generalised Kummer varieties $A^{[[n]]}$ for $n \leq 8$ is included.
\end{abstract}

\section{Introduction}

The two main series of irreducible holomorphic symplectic complex manifolds are Hilbert schemes of points on a K3 surface and generalised Kummer varieties invented by Beauville [1]. One can ask for their complex cobordism class tensored with $\mathbb{Q}$, which is given by the values of all their Chern numbers.

In [6] Ellingsrud, Göttsche and Lehn proved that the complex cobordism class of a Hilbert scheme $X^{[n]}$ of zero-dimensional subschemes of length $n$ on a smooth projective surface $X$ over the complex numbers depends only on the cobordism class of the surface $X$, i.e. on $c_{1}(X)^{2}$ and $c_{2}(X)$ (here and later on, top intersections on surfaces are to be understood as intersection numbers). They showed how this result can be used to calculate the Chern numbers of any such Hilbert scheme $X^{[n]}$ if one knows the Chern numbers of the varieties $\left(\mathbb{P}^{2}\right)^{[k]}$ and $\left(\mathbb{P}^{1} \times \mathbb{P}^{1}\right)^{[k]}$, which in turn can be calculated by means of Bott's residue formula.

Therefore, the Chern numbers of the Hilbert schemes of points on a K3 surface can be efficiently calculated though no explicit formula is known. These numbers can, for example, be used to check the conjecture of [3] about the elliptic genus of the Hilbert schemes $X^{[k]}$ where $X$ is a K3 surface (see [6]). ${ }^{1}$ In this note,

\footnotetext{
Received June 11, 2002.

2000 Mathematics Subject Classification. 14C05, 14M99, 14N10, 14 Q99.

${ }^{1}$ Note added in proof: Borisov and Libgober verified the conjecture of [12] a short time ago, see preprint arXiv:math.AG/0206241
} 
we want to give a method for computing the Chern numbers of the $(n-1)$ dimensional generalised Kummer variety $A^{[[n]]}$ for an abelian surface $A$ and for general $n$ as this has not appeared in the literature so far.

The $\chi_{y^{-}}$genus of $A^{[[n]]}$ has been calculated by Göttsche and Soergel [8]. Expressing this genus in terms of Chern numbers by using the Hirzebruch-RiemannRoch formula gives us enough information to deduce the Chern numbers of $A^{[[n]]}$ for $n \leq 4$.

Using the theory of Rozansky-Witten invariants, Sawon [14] produced a further relation that allowed him to compute all the Chern numbers for $n \leq 5$. The Chern numbers of $A^{[[6]]}$ were calculated by M. Britze and the author in [2]. J. Sawon informed us that he also had computed these numbers. However, all these methods are not sufficient to compute the Chern numbers for $n>6$.

We will give a closed formula describing the value of any complex genus on a generalised Kummer variety in terms of genera of the Hilbert schemes of points on a fixed surface $X$ with $c_{1}(X)^{2} \neq 0$. Since these are computable via Bott's residue formula for $X$ being the projective plane $\mathbb{P}^{2}$, we can compute the Chern numbers of $A^{[[n]]}$ for any $n$. We have done this for $n \leq 11$ (see appendix A, where the values can be found for $n \leq 8)$.

\section{The generalised Kummer varieties}

Let $X$ be a smooth projective surface over the field of complex numbers. For every nonnegative integer we denote by $X^{[n]}$ the Hilbert scheme of zerodimensional subschemes of $X$ of length $n$. By a result of Fogarty [7], this scheme is smooth and projective of dimension $2 n$. It can be viewed as a resolution $\rho: X^{[n]} \rightarrow X^{(n)}$ of the $n$-fold symmetric product $X^{(n)}:=X^{n} / \mathfrak{S}_{n}$ of $X$. The morphism $\rho$, sending closed points, i.e. subschemes of $X$, to their support counting multiplicities, is called the Hilbert-Chow morphism.

Let us briefly recall the construction of the generalised Kummer varieties introduced by Beauville [1]. Let $A$ be an abelian surface and $n>0$. There is an obvious summation morphism $A^{(n)} \rightarrow A$. We denote its composition with the Hilbert-Chow morphism $\rho: A^{[n]} \rightarrow A^{(n)}$ by $\sigma: A^{[n]} \rightarrow A$.

Definition 1. The $n^{\text {th }}$ generalised Kummer variety ${ }^{2} A^{[[n]]}$ is the fibre of $\sigma$ over $0 \in A$.

Beauville showed among other things the following property of the varieties $A^{[[n]]}$ :

Proposition 1. The $n^{\text {th }}$ generalised Kummer variety is a smooth projective holomorphic symplectic variety of dimension $2(n-1)$.

Proof. [1].

\footnotetext{
${ }^{2}$ There is no consistent notation in the literature for what we denote by $A^{[[n]]}$. There are some authors who prefer the symbol $\mathrm{K}^{n-1} A$, and others use $A^{[[n-1]]}$, indicating that this variety is $2(n-1)$-dimensional. However, we believe that for numerical considerations the convention we shall use in this paper is the most convenient.
} 
Let $A$ be an abelian surface again. Since $A$ acts on itself by translation, there is also an induced operation of $A$ on the Hilbert schemes $A^{[n]}$. Let us denote the restriction of this operation to the generalised Kummer variety $A^{[[n]]}$ by $\nu: A \times A^{[n]]} \rightarrow A^{[n]}$. The following diagram is cartesian:

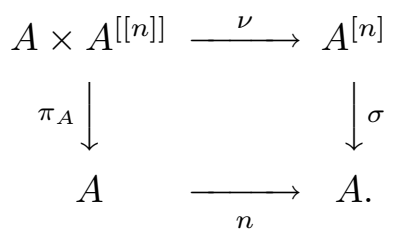

Here, $n: A \rightarrow A, a \mapsto n a$ is the (multiplication by $n$ )-morphism. It is a Galois covering of degree $n^{4}$. Therefore, also $\nu$ is a Galois covering of degree $n^{4}$.

Next, we want to introduce certain line bundles on the Hilbert schemes and generalised Kummer varieties that are constructed from line bundles on the underlying surface:

Each line bundle $L$ on a smooth projective surface $X$ gives us a line bundle $L_{n}$ on $X^{[n]}$ in the following way: $L^{\bigotimes n}$ is a $\mathfrak{S}_{n}$-invariant line bundle on the $n^{\text {th }}$ product $X^{n}$ of $X$. Therefore, we can define the sheaf $L^{(n)}:=\left(\pi_{*}\left(L^{\bigotimes n}\right)\right)^{\mathfrak{S}_{n}}$ of $\mathfrak{S}_{\mathfrak{n}}$-invariant sections of $\pi_{*}\left(L^{\bigotimes n}\right)$ on $X^{(n)}$ where $\pi: X^{n} \rightarrow X^{(n)}$ is the canonical projection. The pull-back $L_{n}:=\rho^{*} L^{(n)}$ by the Hilbert-Chow morphism is a line bundle on $X^{[n]}$. Note that $\operatorname{Pic}(X) \rightarrow \operatorname{Pic}\left(X^{[n]}\right), L \mapsto L_{n}$ is a homomorphism of groups.

This construction has already appeared for example in [6] and [2]. If $X$ is an abelian surface, we denote by $L^{[[n]]}$ the restriction of $L_{n}$ to the generalised Kummer variety $X^{[[n]]} \subseteq X^{[n]}$. By using the seesaw principle (cf. [13]) and $\mathrm{H}^{1}\left(X^{[[n]]}\right)=0$, it can be shown that

$$
\nu^{*} L_{n}=L^{n} \otimes L^{[[n]]}
$$

(cf. [2]).

Besides $L_{n}$, one can define certain further sheaves on $X^{[n]}$ that are constructed from sheaves on $X$. They are called tautological sheaves of $X^{[n]}$ (cf. [11]). The construction is as follows: Since $X^{[n]}$ represents a functor, there is a universal family $\Xi_{n} \subseteq X^{[n]} \times X$ of zero-dimensional subschemes over $X^{[n]}$. Let us denote by $\mathcal{O}_{n}$ its structure sheaf. For any locally free sheaf $F$ of rank $r$ on $X$, we define the sheaf $F^{[n]}:=p_{*}\left(\mathcal{O}_{n} \otimes q^{*} F\right)$ on $X^{[n]}$, where $p: X^{[n]} \times X \rightarrow X^{[n]}$ and $q: X^{[n]} \times X \rightarrow X$ are the canonical projections. $F^{[n]}$ is again locally free of rank $n r$.

The connection between $L_{n}$ and $L^{[n]}$ is as follows: As noted in section 5 of [6], we have

$$
\operatorname{det}\left(F^{[n]}\right)=\operatorname{det}(F)_{n} \otimes\left(\operatorname{det} \mathcal{O}_{X}{ }^{[n]}\right)^{\mathrm{rk} F}
$$

for any locally free sheaf $F$ of constant rank on $X$. Applying this to $F=L$ leads to

$$
L_{n}=\operatorname{det}\left(L^{[n]}\right) \otimes \operatorname{det}\left(\mathcal{O}_{X}^{[n]}\right)^{-1}
$$




\section{Complex genera in general}

Let $\Omega:=\Omega^{\mathrm{U}} \otimes \mathbb{Q}$ denote the (rational) complex cobordism ring. By a result of Milnor $([12])$, it is generated by the cobordism classes $[X]$ of all complex manifolds $X$, and two complex manifolds $X$ and $Y$ lie in the same cobordism class if and only if they have the same Chern numbers, i.e. the Chern numbers determine the cobordism class and vice versa. Recall that the sum in the ring is induced by the disjoint union of manifolds, and the product by the cartesian product of manifolds.

A complex genus $\phi$ is a ring homomorphism $\phi: \Omega \rightarrow R$ into any $\mathbb{Q}$-algebra $R$. By Hirzebruch's theory of genera and multiplicative sequences ([9]), the $R$ valued complex genera are in one-to-one correspondence with the formal power series $f_{\phi} \in R[[x]]$ over $R$ with constant coefficient 1 . The correspondence is given as follows:

$$
\phi(X)=\int_{X} \prod_{i=1}^{n} f_{\phi}\left(\gamma_{i}\right)
$$

for all complex manifolds $X$, where $n$ is the dimension of $X$, and $\gamma_{1}, \ldots, \gamma_{n}$ are the Chern roots of its tangent bundle.

Since the cobordism class is known if one knows the value of all genera (it suffices to know the value of the universal genus $\operatorname{id}_{\Omega}: \Omega \rightarrow \Omega$ ), the knowledge of the values of all genera implies the knowledge of all Chern numbers.

Now, let us slightly generalise the notion of a genus.

Definition 2. Let $\phi$ be a complex genus. For a complex manifold $X$ together with a line bundle $L$ on $X$ we define

$$
\phi(X, L):=\int_{X} e^{c_{1}(L)} \prod_{i=1}^{n} f_{\phi}\left(\gamma_{i}\right)
$$

as the genus $\phi$ of the pair $(X, L)$.

Remark 1. Obviously, $\phi\left(X, \mathcal{O}_{X}\right)=\phi(X)$.

Example 1. If $\operatorname{td}(X)$ denotes the Todd genus of $X$, and $\chi(X, L)$ the holomorphic Euler characteristic of the line bundle $L$ on $X$, we have by the HirzebruchRiemann-Roch theorem that

$$
\operatorname{td}(X, L)=\chi(X, L) .
$$

The genera of pairs $(X, L)$ have the following properties, which follow directly from the appropriate properties of Chern classes/roots.

Proposition 2. Let $\phi: \Omega \rightarrow R$ be any complex genus with values in $R$. We have

1. $\phi(X \times Y, L \otimes M)=\phi(X, L) \cdot \phi(Y, M)$ for two complex manifolds $X$ and $Y$ together with a line bundle $L$ resp. $M$.

2. $\phi\left(X, \nu^{*} L\right)=\operatorname{deg}(\nu) \cdot \phi(Y, L)$ for any Galois covering $\nu: X \rightarrow Y$ and any line bundle $L$ on $Y$. 
Given a genus, we can deform it in the following sense to get a new genus:

Definition 3. Let $\phi$ be a complex genus with values in the ring $R$. By $\phi_{t}$ we denote the genus with values in $R[t]$ given by

$$
\phi_{t}(X):=\int_{X} \prod_{i=1}^{n}\left(f_{\phi}\left(\gamma_{i}\right) e^{t \gamma_{i}}\right)
$$

for any complex manifold $X$.

Remark 2. For any integer $n$, we have $\phi_{n}(X)=\phi\left(X, K_{X}^{-n}\right)$, where $K_{X}$ is the canonical line bundle on $X$.

Example 2. Let $\chi_{y}$ be Hirzebruch's $\chi_{y}$-genus, and $\chi_{y z}$ the twisted $\chi_{y}$-genus (see [15]). In our notation, $\chi_{y z}=\left(\chi_{y}\right)_{z}$.

\section{Complex genera of Hilbert schemes of points on surfaces}

In this section, we want to cite some of the results of [6] and give some corollaries which will be used later on.

Let $X$ be a smooth projective surface. Following [6], we define

$$
H_{X}:=\sum_{n=0}^{\infty}\left[X^{[n]}\right] z^{n}
$$

as an (invertible) element in the formal power series ring $\Omega[[z]]$. Analogously we define

$$
K:=\sum_{n=1}^{\infty}\left[A^{[[n]]}\right] z^{n}
$$

in $\Omega[[z]]$ where $A$ is any abelian surface. The cobordism class does not depend on the choice of $A$ since the generalised Kummer varieties deform with $A$. We can reformulate our task to determine the Chern numbers of the generalised Kummer varieties by asking: What is the value $\phi(K) \in R[[z]]$ for any complex genus $\phi: \Omega \rightarrow R$ ?

The following lemma is a generalization of Theorem 4.2 in [6] for line bundles.

Lemma 1. Let $k$ be a nonnegative integer, $m_{1}, \ldots, m_{k} \in \mathbb{Z}$, and $\phi: \Omega \rightarrow R$ be a genus. Then there exist uniquely determined universal power series $A_{i, j} \in$ $R[[z]], 1 \leq i \leq j \leq k$, and $B_{1}, \ldots, B_{k} \in R[[z]]$, and $C, D \in R[[z]]$ depending only on $\phi$ and $m_{1}, \ldots, m_{k}$ such that for every smooth projective surface $X$, and line bundles $L_{1}, \ldots, L_{k}$ on $X$, we have

$$
\begin{aligned}
& \sum_{n=0}^{\infty} \phi\left(X^{[n]}, \operatorname{det}\left(L_{1}^{[n]}\right)^{m_{1}} \otimes \cdots \otimes \operatorname{det}\left(L_{k}^{[n]}\right)^{m_{k}}\right) z^{n} \\
= & \exp \left(\sum_{1 \leq i \leq j \leq k} c_{1}\left(L_{i}\right) c_{1}\left(L_{j}\right) A_{i j}+\sum_{i=1}^{k} c_{1}\left(L_{i}\right) c_{1}(X) B_{i}+c_{1}(X)^{2} C+c_{2}(X) D\right) .
\end{aligned}
$$


(Remember that top intersection products on surfaces are to be understood as intersection numbers.)

Proof. First note that for $k=1$ the statement of the lemma is just Theorem 4.2 of [6] for the case of line bundles with $\Psi$ (in the notation of [6]) being the Chern character of the $m_{1}^{\text {th }}$ power of the determinant.

Theorem 4.2 of Ellingsrud, Göttsche and Lehn, and the proof presented by them can be easily generalised for more than one bundle, i.e. for $k>1$. Therefore, our lemma as a specialization of this generalization is proven.

From the lemma we conclude the following:

Proposition 3. Let $\phi: \Omega \rightarrow R$ be a genus. Then there exist uniquely determined universal power series $A_{\phi}, B_{\phi}, C_{\phi}, D_{\phi} \in R[[z]]$ depending only on $\phi$ such that for every smooth projective surface $X$ together with a line bundle $L$ on it, we have

$$
\begin{aligned}
\phi\left(H_{X, L}\right) & :=\sum_{n=0}^{\infty} \phi\left(X^{[n]}, L_{n}\right) z^{n} \\
& =\exp \left(c_{1}(L)^{2} A_{\phi}+c_{1}(L) c_{1}(X) B_{\phi}+c_{1}(X)^{2} C_{\phi}+c_{2}(X) D_{\phi}\right) .
\end{aligned}
$$

Proof. We use (4). By the previous lemma,

$$
\begin{aligned}
\phi\left(H_{X, L}\right) & =\sum_{n=0}^{\infty} \phi\left(X^{[n]}, \operatorname{det}\left(L^{[n]}\right) \otimes \operatorname{det}\left(\mathcal{O}_{X}^{[n]}\right)^{-1}\right) z^{n} \\
& =\exp \left(c_{1}(L)^{2} A_{11}+c_{1}(L) c_{1}\left(\mathcal{O}_{X}\right) A_{12}+c_{1}\left(\mathcal{O}_{X}\right)^{2} A_{22}\right. \\
& \left.+c_{1}(L) c_{1}(X) B_{1}+c_{1}\left(\mathcal{O}_{X}\right) c_{1}(X) B_{2}+c_{1}(X)^{2} C+c_{2}(X) D\right)
\end{aligned}
$$

for certain power series $A_{i, j}, B_{i}, C, D$ independent of $X$ and $L$. Since $c_{1}\left(\mathcal{O}_{X}\right)=0$, this proves the proposition with $A_{\phi}=A_{11}, B_{\phi}=B_{1}, C_{\phi}=C$ and $D_{\phi}=D$.

It is possible to express the power series $A_{\phi}$ in terms of genera of Hilbert schemes of points on surfaces:

Proposition 4. Let $\phi: \Omega \rightarrow R$ be any genus. For every smooth projective surface $X$,

$$
c_{1}(X)^{2} A_{\phi}=\frac{1}{2} \ln \frac{\phi_{1}\left(H_{X}\right) \phi_{-1}\left(H_{X}\right)}{\phi\left(H_{X}\right)^{2}} .
$$

Proof. In [10], it is proven that the canonical bundle of $X^{[n]}$ is $K_{n}$ where $K$ denotes the canonical bundle on $X$. It follows $K_{n}^{-m}=\left(K^{-m}\right)_{n}$.

Using this we have by Proposition 3 that

$$
\begin{array}{r}
\ln \phi_{m}\left(H_{X}\right)=\ln \sum_{n=0}^{\infty} \phi_{m}\left(X^{[n]}\right) z^{n}=\ln \sum_{n=0}^{\infty} \phi\left(X^{[n]}, K_{n}^{-m}\right) z^{n} \\
=m^{2} A_{\phi} c_{1}(K)^{2}-m B_{\phi} c_{1}(K) c_{1}(X)+C_{\phi} c_{1}(X)^{2}+D_{\phi} c_{2}(X) \\
=\left(m^{2} A_{\phi}+m B_{\phi}+C_{\phi}\right) c_{1}(X)^{2}+D_{\phi} c_{2}(X)
\end{array}
$$


for all integers $m$, which proves the proposition.

\section{Complex genera of the generalised Kummer varieties}

In this section, we will relate the (generalised) complex genera of Beauville's generalised Kummer varieties to the complex genera of Hilbert schemes of points on a surface, which we studied in the previous section.

The first step in this direction is the following:

Proposition 5. Let $\phi: \Omega \rightarrow R$ be a complex genus with values in the $\mathbb{Q}$-algebra $R$. For every abelian surface $A$ together with a line bundle $L$ on it, we have

$$
c_{1}(L)^{2} \phi\left(A^{[[n]]}, L^{[[n]]}\right)=2 n^{2} \phi\left(A^{[n]}, L_{n}\right)
$$

for all positive integers $n$.

Proof. We will make use of (2). Recall that $\nu$ is a Galois covering of degree $n^{4}$. By Proposition 2 we have

$$
\begin{aligned}
\phi\left(A, L^{n}\right) \phi\left(A^{[[n]]}, L^{[[n]]}\right)=\phi(A \times & \left.A^{[[n]]}, L^{n} \otimes L^{[[n]]}\right) \\
& =\phi\left(A \times A^{[[n]]}, \nu^{*} L_{n}\right)=n^{4} \phi\left(A^{[n]}, L_{n}\right),
\end{aligned}
$$

which proves the proposition, once we have shown that $\phi\left(A, L^{n}\right)=\frac{n^{2}}{2} c_{1}(L)^{2}$. This follows from the fact that the Chern classes of an abelian surface are trivial:

$$
\phi\left(A, L^{n}\right)=\int_{A} f_{\phi}\left(\gamma_{1}\right) f_{\phi}\left(\gamma_{2}\right) e^{c_{1}\left(L^{n}\right)}=\int_{A} \frac{c_{1}\left(L^{n}\right)^{2}}{2}=\frac{n^{2}}{2} c_{1}(L)^{2},
$$

where we have used that $f_{\phi}$ is a power series with constant coefficient 1 .

In [2], M. Britze and the author expressed the (holomorphic) Euler characteristic of the line bundle $L^{[[n]]}$ in terms of the Euler characteristic of $L$ in order to deduce a formula for the Euler characteristic of an arbitrary line bundle $M$ on $A^{[[n]]}$ as a polynomial in the Beauville-Bogomolov quadratic form of $c_{1}(M)$. By using the analogous expression of the Euler characteristic of the line bundle $L_{n}$ on $A^{[n]}$ (see [6]), we get the mentioned result of [2] as a corollary of the previous proposition:

Corollary 1 ([2]). The holomorphic Euler characteristic of the line bundle $L^{[[n]]}$ on $A^{[[n]]}$ is given by

$$
\chi\left(A^{[[n]]}, L^{[[n]]}\right)=n\left(\begin{array}{c}
\chi(A, L)+n-1 \\
n-1
\end{array}\right) .
$$

Proof. By Lemma 5.1 of [6] we have

$$
\chi\left(A^{[n]}, L_{n}\right)=\left(\begin{array}{c}
\chi(A, L)+n-1 \\
n
\end{array}\right) .
$$

Using this, the corollary follows from the proposition applied to the case for $\phi$ being the Todd genus (remember Example 1). Also note that $\chi(A, L)=\frac{1}{2} c_{1}(L)^{2}$ by the Hirzebruch-Riemann-Roch formula. 
If we are interested in the usual genera of the generalised Kummer varieties, i.e. the genera of the pairs $\left(A^{[[n]]}, \mathcal{O}_{A[[n]]}\right)$, we cannot use Proposition 5 directly since for $L=\mathcal{O}_{A}$ it just states $0=0$.

However, it is still possible to make use of the proposition. We have to look at all generalised Kummer varieties at the same time. Doing so, we get the following main result of this work:

Theorem 1. ${ }^{3}$ Let $\phi: \Omega \rightarrow R$ be a complex genus with values in the $\mathbb{Q}$-algebra $R$. For every smooth projective surface $X$ with $\int_{X} c_{1}(X)^{2} \neq 0$,

$$
\phi(K)=\frac{1}{c_{1}(X)^{2}}\left(z \frac{\mathrm{d}}{\mathrm{d} z}\right)^{2} \ln \frac{\phi_{1}\left(H_{X}\right) \phi_{-1}\left(H_{X}\right)}{\phi\left(H_{X}\right)^{2}} .
$$

Proof. Let $L$ be any line bundle on $A$. We have

$$
\begin{gathered}
c_{1}(L)^{2} \sum_{n=1}^{\infty} \phi\left(A^{[[n]]}, L^{[[n]]}\right) z^{n}=2 \sum_{n=1}^{\infty} n^{2} \phi\left(A^{[n]}, L_{n}\right) z^{n}=2\left(z \frac{\mathrm{d}}{\mathrm{d} z}\right)^{2} \phi\left(H_{A, L}\right) \\
=2\left(z \frac{\mathrm{d}}{\mathrm{d} z}\right)^{2} \exp \left(c_{1}(L)^{2} A_{\phi}+c_{1}(L) c_{1}(A) B_{\phi}+c_{1}(A)^{2} C_{\phi}+c_{2}(A) D_{\phi}\right) \\
=2\left(z \frac{\mathrm{d}}{\mathrm{d} z}\right)^{2} \exp \left(c_{1}(L)^{2} A_{\phi}\right)=2\left(z \frac{\mathrm{d}}{\mathrm{d} z}\right)^{2} c_{1}(L)^{2} A_{\phi}+\mathrm{O}\left(\left(c_{1}(L)^{2}\right)^{2}\right)
\end{gathered}
$$

which together with Proposition 4 proves the theorem, since there are line bundles on $A$ with $c_{1}(L) \neq 0$.

Let $\operatorname{id}_{\Omega}: \Omega \rightarrow \Omega$ be the universal genus. Like any other genus, we can deform it to a genus $\left(\operatorname{id}_{\Omega}\right)_{t}: \Omega \rightarrow \Omega[t]$. If $X \in \Omega$ is a rational complex cobordism class, we write

$$
X_{t}:=\left(\operatorname{id}_{\Omega}\right)_{t}(X) \in \Omega[t] .
$$

With this notation, we can express the cobordism class $K$ by

$$
K=\frac{1}{c_{1}(X)^{2}}\left(z \frac{\mathrm{d}}{\mathrm{d} z}\right)^{2} \ln \frac{\left(H_{X}\right)_{1}\left(H_{X}\right)_{-1}}{H_{X}^{2}}
$$

for all surfaces $X$ with $c_{1}(X)^{2} \neq 0$.

Remark 3. Of course, everything in this chapter still holds true if we replace the abelian surfaces $A$ from which we constructed the generalised Kummer varieties, by an arbitrary complex torus of dimension two.

We used Theorem 1 to produce a table of all Chern numbers of the generalised Kummer varieties up to dimension fourteen. It can be found in Appendix A.

\footnotetext{
${ }^{3}$ Note added in proof: the author has recently managed to use this formula to compute the elliptic genera of generalised Kummer varieties, see preprint arXiv:math.AG/0208077
} 


\section{Appendix A. The Chern numbers of the generalised Kummer varieties of dimension up to 14}

We have used Theorem 1 to compute all Chern numbers of the generalised Kummer varieties of dimension up to fourteen. Our results are as follows:

\begin{tabular}{|c|c|c|c|}
\hline Chern number & Evaluated on $A^{[[*]]}$ & Chern number & Evaluated on $A^{[[*]]}$ \\
\hline$c_{2}$ & 24 & $c_{2}^{7}$ & 421414305792 \\
\hline$c_{2}^{2}$ & 756 & $c_{2}^{5} c_{4}$ & 149664301056 \\
\hline$c_{4}$ & 108 & $c_{2}^{3} c_{4}^{2}$ & 53149827072 \\
\hline$c_{2}^{3}$ & 30208 & $c_{2} c_{4}^{3}$ & 18874417152 \\
\hline$c_{2} c_{4}$ & 6784 & $c_{2}^{4} c_{6}$ & 24230756352 \\
\hline$c_{6}$ & 448 & $c_{2}^{2} c_{4} c_{6}$ & 8610545664 \\
\hline$c_{2}^{4}$ & 1470000 & $c_{4}^{2} c_{6}$ & 3059945472 \\
\hline$c_{2}^{2} c_{4}$ & 405000 & $c_{2} c_{6}^{2}$ & 1397121024 \\
\hline$c_{4}^{2}$ & 111750 & $c_{2}^{3} c_{8}$ & 1914077184 \\
\hline$c_{2} c_{6}$ & 37500 & $c_{2} c_{4} c_{8}$ & 681332736 \\
\hline$c_{8}$ & 750 & $c_{6} c_{8}$ & 110853120 \\
\hline$c_{2}^{5}$ & 84478464 & $c_{2}^{2} c_{10}$ & 71909376 \\
\hline$c_{2}^{3} c_{4}$ & 26220672 & $c_{4} c_{10}$ & 25700352 \\
\hline$c_{2} c_{4}^{2}$ & 8141472 & $c_{2} c_{12}$ & 1198080 \\
\hline$c_{2}^{2} c_{6}$ & 3141504 & $c_{14}$ & 7680 \\
\hline$c_{4} c_{6}$ & 979776 & & \\
\hline$c_{2} c_{8}$ & 142560 & & \\
\hline$c_{10}$ & 2592 & & \\
\hline$c_{2}^{6}$ & 5603050432 & & \\
\hline$c_{2}^{4} c_{4}$ & 1881462016 & & \\
\hline$c_{2}^{2} c_{4}^{2}$ & 631808744 & & \\
\hline$c_{4}^{3}$ & 212190776 & & \\
\hline$c_{2}^{3} c_{6}$ & 268796752 & & \\
\hline$c_{2} c_{4} c_{6}$ & 90412056 & & \\
\hline$c_{6}^{2}$ & 12976376 & & \\
\hline$c_{2}^{2} c_{8}$ & 17075912 & & \\
\hline$c_{4} c_{8}$ & 5762400 & & \\
\hline$c_{2} c_{10}$ & 441784 & & \\
\hline$c_{12}$ & 2744 & & \\
\hline
\end{tabular}

It is a remarkable fact that all Chern numbers of the varieties $A^{[[n]]}$ with $n \leq 8$ are positive and divisible by $n^{3}$. (This result is also true for $n \leq 11$.) As the known Chern numbers of Hilbert schemes of points on K3 surfaces are also positive one can wonder if, given a compact Hyperkähler manifold $X$, all Chern numbers of $X$ are positive. 


\section{Acknowledgements}

I would like to thank Michael Britze and Daniel Huybrechts who discussed the ideas presented in this note with me. Without them, it would have taken much more time to complete this paper.

\section{References}

[1] A. Beauville, Variétés Kähleriennes dont la première classe de Chern est nulle, J. Differential Geom. 18 (1983), 755-782.

[2] M. Britze, M. A. Nieper, Hirzebruch-Riemann-Roch formulae on Irreducible Symplectic Kähler Manifolds, preprint; arXiv:math.AG/0101062

[3] R. Dijkgraaf, G. Moore, E. Verlinde, H. Verlinde, Elliptic genera of symmetric products and second quantized strings, Comm. Math. Phys. 185 (1997), 197-201.

[4] G. Ellingsrud, S. A. Strømme, On the homology of the Hilbert scheme of points in the plane, Invent. Math. 87 (1987), 343-352.

[5] , Bott's formula and enumerative geometry, J. Amer. Math. Soc. 9 (1996), 175193.

[6] G. Ellingsrud, L. Göttsche, M. Lehn, On the cobordism class of the Hilbert scheme of a surface, J. Algebraic Geom. 10 (2001), 81-100.

[7] J. Fogarty, Algebraic families on an algebraic surface, Amer. J. Math. 90 (1968), 511-521.

[8] L. Göttsche, W. Soergel, Perverse sheaves and the cohomology of Hilbert schemes of smooth algebraic surfaces, Math. Ann. 296 (1993), 235-245.

[9] F. Hirzebruch, Neue topologische Methoden in der algebraischen Geometrie, Zweite ergänzte Auflage. Ergebnisse der Mathematik und ihrer Grenzgebiete, N.F., Heft 9 Springer-Verlag, Berlin-Göttingen-Heidelberg, 1962.

[10] D. Huybrechts, M. Lehn, The geometry of moduli spaces of sheaves, Aspects of Mathematics, E31. Friedr. Vieweg \& Sohn, Braunschweig, 1997.

[11] M. Lehn, Chern classes of tautological sheaves on Hilbert schemes of points on surfaces, Invent. Math. 136 (1999), 157-207.

[12] J. Milnor, On the cobordism ring $\Omega^{*}$ and a complex analogue. I. Amer. J. Math. 82 (1960), 505-521.

[13] D. Mumford, Abelian varieties, Tata Institute of Fundamental Research Studies in Mathematics, No. 5. Oxford University Press, London, 1970.

[14] J. Sawon, Rozansky-Witten invariants of hyperkähler manifolds, PhD thesis, University of Cambridge, 1999.

[15] B. Totaro, Chern numbers for singular varierties and elliptic homology, Ann. of Math. (2) 151 (2000), 757-791.

Mathematisches Institut Der Univ. Zu Köln, Weyertal 86-90, 0931 Köln, Germany. E-mail address: mnieper@mi.uni-koeln.de 